\title{
Performance of the Vibrational Self-Consistent Field Theory for the Accurate Potential Energy Surfaces: Fundamentals, Excited States and Intensities
}

\author{
Tapta Kanchan Roy* \\ Department of Chemistry and Chemical Sciences,
}

Central University of Jammu, Rahya-Suchani (Bagla), Dist: Samba, Jammu and Kashmir,

181143, India

email: tapta.che@cujammu.ac.in

\begin{tabular}{|c|c|c|}
\hline S1. No & Contents & Page No. \\
\hline 1 & Fig. S1 Molecular structures under study & 2 \\
\hline 2 & $\begin{array}{l}\text { Table } \mathrm{S} 1 \text {. Comparison of the degenerate perturbation theory with VSCF and VSCF- } \\
\text { PT2 theory for } \mathrm{H}_{2} \mathrm{O} \text { and } \mathrm{H}_{2} \mathrm{CO} \text { molecules (in } \mathrm{cm}^{-1} \text { ) }\end{array}$ & 3 \\
\hline 3 & $\begin{array}{l}\text { Table S2. Comparisons of harmonic (HO), VSCF and VSCF-PT2 values with } \\
\text { experiment for fundamental transitions (in } \mathrm{cm}^{-1} \text { ) computed at CCSD and CCSD(T) } \\
\text { potentials for pentaomic molecules along with the statistical error analysis }\end{array}$ & 4 \\
\hline 4 & $\begin{array}{l}\text { Table S3. Comparisons of harmonic (HO), VSCF and VSCF-PT2 values with } \\
\text { experiment for fundamental transitions (in } \mathrm{cm}^{-1} \text { ) computed at CCSD and CCSD(T) } \\
\text { potentials for hexatomic molecules along with the statistical error analysis }\end{array}$ & $5-6$ \\
\hline 5 & $\begin{array}{l}\text { Table S4. Comparisons of harmonic (HO), VSCF and VSCF-PT2 values with } \\
\text { experiment for fundamental transitions (in } \mathrm{cm}^{-1} \text { ) computed at CCSD and CCSD(T) } \\
\text { potentials for heptatomic molecules along with the statistical error analysis }\end{array}$ & $7-8$ \\
\hline 6 & $\begin{array}{l}\text { Table S5. Comparison of different vibrational algorithms for the anharmonic } \\
\text { transitions of } \mathrm{H}_{2} \mathrm{CO}\left(\mathrm{in} \mathrm{cm}^{-1}\right)\end{array}$ & 9 \\
\hline 7 & $\begin{array}{l}\text { Fig. S2. Comparisons of the errors of VSCF-PT2 and VCI. In the left and right } \\
\text { panels the reference values are experiment and VCI, respectively. Here VCI is } \\
\text { VCI(SDTQ). }\end{array}$ & 9 \\
\hline 8 & $\begin{array}{l}\text { Table S6. Comparisons of harmonic (HO), VSCF and VSCF-PT2 values with } \\
\text { experiment for the fundamental transitions }\left(\mathrm{in}^{-1} \mathrm{~cm}^{-1} \text { computed at different CC level }\right. \\
\text { of theories along with the statistical error analysis. }\end{array}$ & 10 \\
\hline 9 & $\begin{array}{l}\text { Table S7. Comparisons of the VSCF-PT2 fundamental transitions (in } \mathrm{cm}^{-1} \text { ) } \\
\text { computed at } \mathrm{CCSD}(\mathrm{T}) \text { with different Dunning basis for } \mathrm{H}_{2} \mathrm{O}, \mathrm{H}_{2} \mathrm{CO} \text { and } \mathrm{HCOOH} \\
\text { along the statistical error analysis. }\end{array}$ & 11 \\
\hline 10 & $\begin{array}{l}\text { Table S8. Comparisons of the VSCF-PT2 values computed at CCSD(T) method } \\
\text { using pure and hybrid (cc-pVQZ /cc-pVTZ) PES with experimental values }\left(\text { in } \mathrm{cm}^{-1} \text { ) }\right.\end{array}$ & 12 \\
\hline 11 & S9. Cartesian coordinates of equilibrium geometries of some selected molecules & $13-14$ \\
\hline 12 & S10. Source and references of experimental data for each molecule & 15 \\
\hline
\end{tabular}



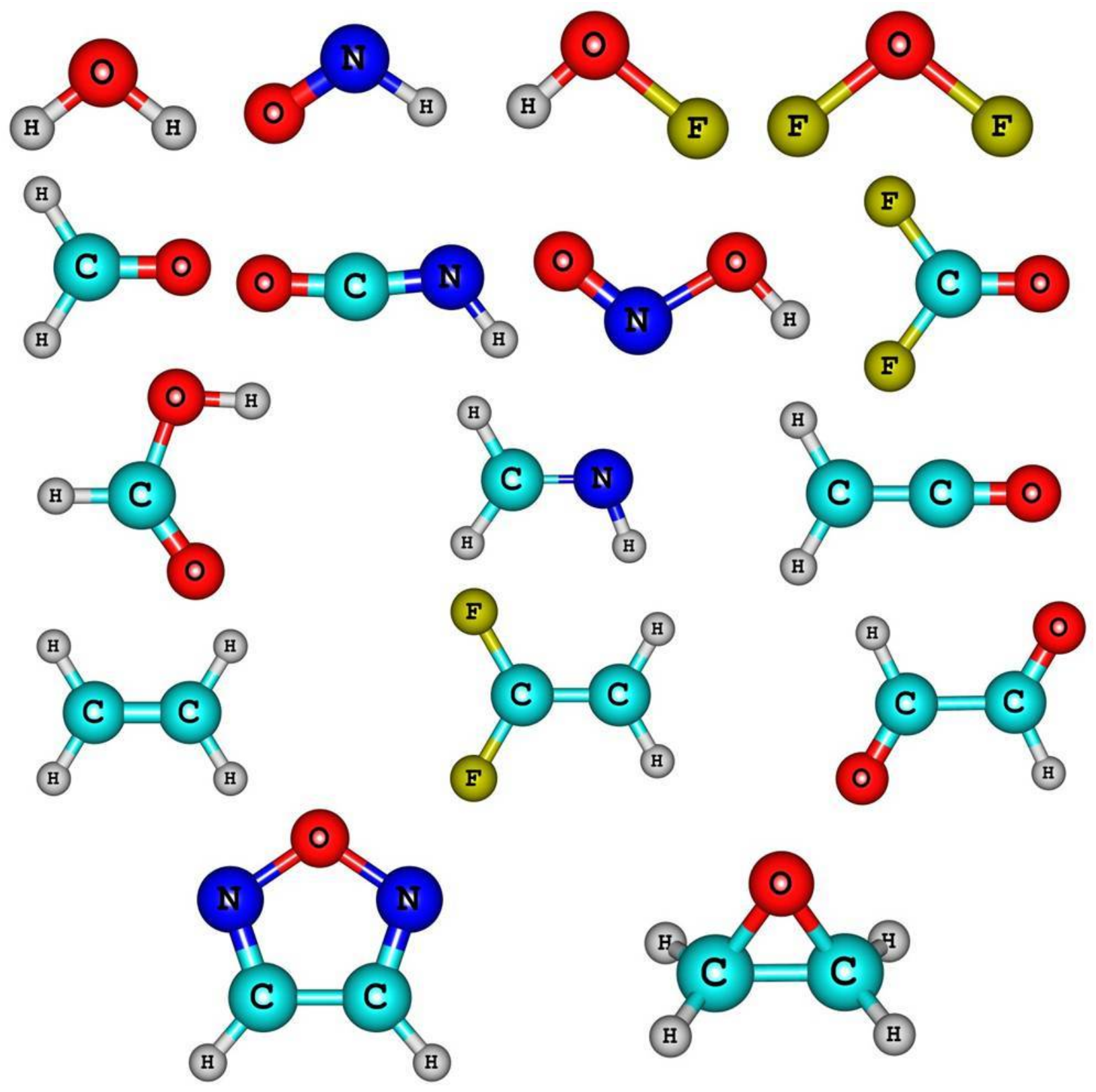

Fig S1. Molecular structures under study 
Table S1. Comparison of the degenerate perturbation theory (DPT2-VSCF) and VSCF-PT2 for $\mathrm{H}_{2} \mathrm{O}$ and $\mathrm{H}_{2} \mathrm{CO}$ molecules at CCSD(T)/cc-pVTZ level of theory (in $\mathrm{cm}^{-1}$ )

\begin{tabular}{|c|c|c|}
\hline $\mathbf{H}_{\mathbf{2}} \mathbf{O}$ & VSCF-PT2 & DPT2-VSCF \\
\hline$v_{1}$ & 3725.68 & 3725.68 \\
\hline$v_{2}$ & 3660.64 & 3660.65 \\
\hline$v_{3}$ & 1602.59 & 1602.60 \\
\hline $\mathbf{H}_{2} \mathbf{C O}$ & VSCF-PT2 & DPT2-VSCF \\
\hline$v_{1}$ & 2773.40 & 2773.40 \\
\hline$v_{2}$ & 2766.66 & 2766.7 \\
\hline$v_{3}$ & 1749.33 & 1749.5 \\
\hline$v_{4}$ & 1504.73 & 1504.8 \\
\hline$v_{5}$ & 1246.86 & 1246.9 \\
\hline$v_{6}$ & 1155.23 & 1155.2 \\
\hline
\end{tabular}


Table S2. Comparisons of harmonic (HO), VSCF and VSCF-PT2 values with experiment for fundamental transitions $\left(\right.$ in $\mathrm{cm}^{-1}$ ) computed at $\mathrm{CCSD}$ and $\operatorname{CCSD}(\mathrm{T})$ potentials for pentatomic molecules along with the statistical error analysis

\begin{tabular}{|c|c|c|c|c|c|c|c|c|}
\hline & Mode type & & \multicolumn{3}{|c|}{ CCSD } & \multicolumn{3}{|c|}{ CCSD(T) } \\
\hline & НСOOH & $\operatorname{Exp}$ & HO & VSCF & VSCF-PT2 & HO & VSCF & VSCF-PT2 \\
\hline$v_{1}$ & $\mathrm{OH}$ str & 3570 & 3813.9 & 3563.5 & 3602.6 & 3763.3 & 3508.6 & 3547.1 \\
\hline$v_{2}$ & $\mathrm{CH}$ str & 2943 & 3116.7 & 2941.5 & 2966.4 & 3089.2 & 2909.0 & 2932.5 \\
\hline$v_{3}$ & $\mathrm{C}=\mathrm{O}$ str & 1770 & 1868.1 & 1838.3 & 1835.0 & 1824.6 & 1793.1 & 1789.5 \\
\hline$v_{4}$ & $\mathrm{CH}$ bend & 1387 & 1435.8 & 1403.0 & 1397.2 & 1415.7 & 1381.5 & 1375.5 \\
\hline$v_{5}$ & $\mathrm{OH}$ bend & 1229 & 1344.7 & 1312.5 & 1304.2 & 1326.0 & 1292.7 & 1284.6 \\
\hline$v_{6}$ & C-O str & 1105 & 1162.0 & 1135.7 & 1116.3 & 1137.1 & 1109.6 & 1090.4 \\
\hline$v_{7}$ & $\mathrm{CH}$ bend & 1033 & 1084.4 & 1062.2 & 1056.2 & 1061.0 & 1037.2 & 1030.9 \\
\hline$v_{8}$ & Torsion & 638 & 676.3 & 657.8 & 597.2 & 676.6 & 657.4 & 598.2 \\
\hline \multirow[t]{5}{*}{$v_{9}$} & OCO deform & 625 & 642.4 & 640.0 & 631.7 & 629.4 & 626.2 & 618.0 \\
\hline & MaxAD & & 243.9 & 83.5 & 75.2 & 193.3 & 63.7 & 55.6 \\
\hline & MAD & & 93.8 & 30.1 & 32.0 & 69.2 & 24.1 & 20.4 \\
\hline & MAPE & & 5.57 & 2.57 & 2.55 & 3.98 & 1.53 & 1.82 \\
\hline & $\mathrm{H}_{2} \mathrm{CCO}$ & & & & & & & \\
\hline$v_{1}$ & $\mathrm{CH} 2$ a-str & 3165 & 3328.9 & 3140.2 & 3106.9 & 3305.4 & 3109.0 & 3074.1 \\
\hline$v_{2}$ & $\mathrm{CH} 2$ s-str & 3070 & 3224.0 & 3107.2 & 3076.2 & 3201.0 & 3077.9 & 3043.0 \\
\hline$v_{3}$ & $\mathrm{C}=\mathrm{O}$ stretch & 2153 & 2236.2 & 2197.0 & 2193.3 & 2196.7 & 2153.7 & 2149.5 \\
\hline$v_{4}$ & CH2 scissor & 1387 & 1442.2 & 1422.8 & 1435.0 & 1419.6 & 1396.2 & 1399.0 \\
\hline$v_{5}$ & $\mathrm{C}=\mathrm{C}$ stretch & 1116 & 1175.6 & 1164.4 & 1163.5 & 1151.8 & 1137.6 & 1133.6 \\
\hline$v_{6}$ & $\mathrm{CH} 2$ rock & 978 & 1011.6 & 1007.5 & 1003.3 & 992.3 & 986.8 & 982.4 \\
\hline$v_{7}$ & $\mathrm{CH} 2$ wag & 587 & 612.7 & 617.6 & 585.8 & 590.1 & 595.7 & 562.8 \\
\hline$v_{8}$ & $\mathrm{C}=\mathrm{C}=\mathrm{O}$ bend & 528 & 549.6 & 549.7 & 517.9 & 514.6 & 516.4 & 464.8 \\
\hline \multirow[t]{5}{*}{$v_{9}$} & $\mathrm{C}=\mathrm{C}=\mathrm{O}$ bend & 439 & 447.1 & 464.5 & 448.5 & 432.3 & 449.6 & 432.5 \\
\hline & MaxAD & & 163.5 & 48.4 & 58.5 & 140.0 & 56.4 & 91.3 \\
\hline & MAD & & 67.2 & 33.1 & 27.5 & 46.8 & 15.1 & 27.9 \\
\hline & MAPE & & 4.12 & 3.23 & 2.08 & 2.49 & 1.31 & 2.73 \\
\hline & $\mathrm{CH}_{2} \mathrm{NH}$ & & & & & & & \\
\hline$v_{1}$ & NH stretch & 3263 & 3480.9 & 3267.5 & 3289.1 & 3440.6 & 3222.1 & 3243.4 \\
\hline$v_{2}$ & $\mathrm{CH}$ a. stretch & 3024 & 3172.6 & 2990.0 & 2973.4 & 3152.4 & 2966.2 & 2948.6 \\
\hline$v_{3}$ & CH s. stretch & 2914 & 3076.0 & 2936.1 & 2906.7 & 3052.2 & 2907.1 & 2877.7 \\
\hline$v_{4}$ & $\mathrm{C}=\mathrm{N}$ stretch & 1638 & 1717.4 & 1683.9 & 1681.1 & 1675.3 & 1639.0 & 1636.0 \\
\hline$v_{5}$ & $\mathrm{CH}_{2}$ scissors & 1452 & 1504.3 & 1477.5 & 1471.6 & 1483.2 & 1455.7 & 1449.1 \\
\hline$v_{6}$ & HCNH deform & 1344 & 1400.4 & 1369.6 & 1365.8 & 1386.9 & 1355.2 & 1351.3 \\
\hline$v_{7}$ & Torsion & 1127 & 1179.3 & 1142.1 & 1134.6 & 1161.8 & 1123.7 & 1116.1 \\
\hline$v_{8}$ & $\mathrm{H}_{2} \mathrm{CN}$ deform & 1061 & 1105.5 & 1080.0 & 1074.2 & 1081.1 & 1054.8 & 1048.7 \\
\hline \multirow[t]{4}{*}{$v_{9}$} & HCNH deform & 1058 & 1085.7 & 1075.8 & 1067.4 & 1073.0 & 1062.3 & 1054.0 \\
\hline & MaxAD & & 217.9 & 45.9 & 50.6 & 177.6 & 57.8 & 75.4 \\
\hline & MAD & & 93.5 & 23.3 & 22.1 & 69.5 & 15.0 & 19.0 \\
\hline & MAPE & & 4.58 & 1.48 & 1.24 & 3.16 & 0.65 & 0.86 \\
\hline
\end{tabular}


Table S3. Comparisons of harmonic (HO), VSCF and VSCF-PT2 values with experiment for fundamental transitions (in $\mathrm{cm}^{-1}$ ) computed at $\operatorname{CCSD}$ and $\operatorname{CCSD}(\mathrm{T})$ potentials for thexatomic molecules along with the statistical error analysis

\begin{tabular}{|c|c|c|c|c|c|c|c|c|}
\hline & Mode type & & \multicolumn{3}{|c|}{ CCSD } & \multicolumn{3}{|c|}{$\operatorname{CCSD}(\mathrm{T})$} \\
\hline & $\mathrm{C}_{2} \mathrm{H}_{4}$ & Exp & HO & VSCF & VSCF-PT2 & HO & VSCF & VSCF-PT2 \\
\hline$v_{1}$ & CH2 a-str & 3106 & 3264.9 & 3134.2 & 3120.4 & 3246.3 & 3113.1 & 3099.1 \\
\hline$v_{2}$ & $\mathrm{CH} 2$ a-str & 3103 & 3238.3 & 3106.8 & 3093.0 & 3219.4 & 3085.4 & 3071.0 \\
\hline$v_{3}$ & $\mathrm{CH} 2 \mathrm{~s}-\mathrm{str}$ & 3026 & 3178.5 & 3083.9 & 3030.5 & 3157.2 & 3060.3 & 3004.4 \\
\hline$v_{4}$ & $\mathrm{CH} 2 \mathrm{~s}-\mathrm{str}$ & 2989 & 3159.0 & 3043.4 & 3031.1 & 3139.2 & 3021.2 & 3008.6 \\
\hline$v_{5}$ & CC str & 1623 & 1703.3 & 1671.8 & 1664.5 & 1671.6 & 1638.0 & 1630.5 \\
\hline$v_{6}$ & $\mathrm{CH} 2$ scis & 1444 & 1493.4 & 1465.0 & 1463.2 & 1478.8 & 1450.0 & 1447.7 \\
\hline$v_{7}$ & $\mathrm{CH} 2$ scis & 1342 & 1387.7 & 1368.3 & 1362.0 & 1368.9 & 1348.1 & 1341.6 \\
\hline$v_{8}$ & $\mathrm{CH} 2$ rock & 1236 & 1255.4 & 1242.0 & 1240.1 & 1242.0 & 1228.4 & 1226.4 \\
\hline$v_{9}$ & $\mathrm{CH} 2$ twist & 1023 & 1063.9 & 1048.2 & 1044.1 & 1047.0 & 1030.7 & 1026.5 \\
\hline$v_{10}$ & $\mathrm{CH} 2$ wag & 949 & 986.7 & 980.00 & 973.9 & 966.7 & 960.2 & 953.8 \\
\hline$v_{11}$ & $\mathrm{CH} 2$ wag & 943 & 971.2 & 964.7 & 959.5 & 942.0 & 936.5 & 930.9 \\
\hline$v_{12}$ & $\mathrm{CH} 2$ rock & 826 & 832.6 & 845.9 & 838.3 & 822.7 & 835.6 & 827.9 \\
\hline & MaxAD & & 170.0 & 57.9 & 42.1 & 150.2 & 34.3 & 32.0 \\
\hline & MAD & & 77.1 & 28.7 & 19.3 & 58.4 & 13.4 & 10.3 \\
\hline & MAPE & & 3.78 & 1.84 & 1.33 & 2.52 & 0.77 & 0.54 \\
\hline & $\mathrm{C}_{2} \mathrm{H}_{2} \mathrm{O}_{2}$ & & & & & & & \\
\hline$v_{1}$ & $\mathrm{CH}$ str & 2843 & 3020.3 & 2885.5 & 2858.5 & 2987.5 & 2848.3 & 2817.9 \\
\hline$v_{2}$ & $\mathrm{CH}$ str & 2835 & 3016.3 & 2823.1 & 2789.4 & 2982.6 & 2782.7 & 2747.7 \\
\hline$v_{3}$ & CO str & 1745 & 1841.9 & 1817.0 & 1811.7 & 1780.0 & 1753.2 & 1749.1 \\
\hline$v_{4}$ & CO str & 1732 & 1814.8 & 1789.5 & 1784.5 & 1758.0 & 1730.7 & 1725.5 \\
\hline$v_{5}$ & $\mathrm{CH}$ bend & 1338 & 1405.1 & 1378.4 & 1376.0 & 1385.0 & 1357.2 & 1354.6 \\
\hline$v_{6}$ & $\mathrm{CH}$ bend & 1312 & 1361.9 & 1336.7 & 1333.4 & 1342.4 & 1315.7 & 1312.4 \\
\hline$v_{7}$ & $\mathrm{CC}$ str & 1065 & 1113.5 & 1086.9 & 1081.3 & 1095.5 & 1067.2 & 1061.0 \\
\hline$v_{8}$ & $\mathrm{CH}$ bend & 1048 & 1093.2 & 1086.8 & 1083.1 & 1068.4 & 1061.0 & 1057.0 \\
\hline$v_{9}$ & $\mathrm{CH}$ bend & 801 & 838.1 & 837.8 & 825.7 & 823.7 & 821.8 & 809.4 \\
\hline$v_{10}$ & CCO deform & 551 & 570.5 & 562.8 & 562.2 & 560.2 & 551.6 & 551.01 \\
\hline$v_{11}$ & CCO deform & 339 & 339.3 & 346.1 & 341.3 & 330.5 & 337.3 & 332.5 \\
\hline $\mathrm{v}_{12}$ & Torsion & 127 & 135.0 & 155.8 & 141.8 & 133.9 & 153.0 & 139.6 \\
\hline & MaxAD & & 181.3 & 72.0 & 66.7 & 147.6 & 52.3 & 87.3 \\
\hline & MAD & & 67.8 & 32.8 & 28.7 & 44.0 & 12.9 & 15.0 \\
\hline & MAPE & & 4.60 & 4.29 & 2.99 & 3.07 & 2.45 & 1.66 \\
\hline
\end{tabular}




\begin{tabular}{|r|l|r|r|r|r|r|r|r|}
\hline \multicolumn{1}{|c|}{$\mathbf{C}_{2} \mathbf{F}_{2} \mathbf{H}_{\mathbf{2}}$} & \multicolumn{7}{|c|}{} \\
\hline$v_{1}$ & CH2 a-str & $\mathbf{3 1 7 6}$ & 3336.1 & 3134.8 & 3101.9 & 3316.8 & 3111.9 & 3078.3 \\
\hline$v_{2}$ & CH2 s-str & $\mathbf{3 0 5 8}$ & 3231.2 & 3101.9 & 3079.5 & 3210.4 & 3078.7 & 3055.3 \\
\hline$v_{3}$ & CC str & $\mathbf{1 7 2 9}$ & 1815.8 & 1785.1 & 1776.9 & 1778.1 & 1745.7 & 1737.4 \\
\hline$v_{4}$ & CH2 scis & $\mathbf{1 3 5 9}$ & 1438.4 & 1413.3 & 1404.7 & 1422.3 & 1396.3 & 1386.8 \\
\hline$v_{5}$ & H2CC Deform & $\mathbf{1 3 0 1}$ & 1378.9 & 1355.6 & 1352.0 & 1354.1 & 1330.3 & 1326.6 \\
\hline$v_{6}$ & CH2 twist & $\mathbf{9 5 4}$ & 986.1 & 983.2 & 976.4 & 972.5 & 969.2 & 962.3 \\
\hline$v_{7}$ & CF2 str & $\mathbf{9 2 6}$ & 960.4 & 953.0 & 947.5 & 942.4 & 934.4 & 928.9 \\
\hline$v_{8}$ & CH2 twist & $\mathbf{8 0 2}$ & 847.3 & 859.0 & 843.6 & 815.3 & 828.8 & 812.1 \\
\hline$v_{9}$ & CH2 rock & $\mathbf{7 0 8}$ & 737.8 & 752.6 & 734.9 & 723.6 & 738.9 & 720.6 \\
\hline$v_{10}$ & Torsion & $\mathbf{6 1 0}$ & 643.3 & 635.6 & 634.3 & 624.4 & 615.0 & 614.0 \\
\hline$v_{11}$ & CF2 bend & $\mathbf{5 5 0}$ & 563.4 & 559.2 & 559.0 & 554.9 & 550.4 & 550.1 \\
\hline$v_{12}$ & CH2 torsion & $\mathbf{4 3 7}$ & 445.0 & 456.3 & 445.7 & 437.6 & 448.6 & 437.7 \\
\hline & MaxAD & & $\mathbf{1 7 3 . 2}$ & $\mathbf{5 7 . 0}$ & $\mathbf{7 4 . 2}$ & $\mathbf{1 5 2 . 4}$ & $\mathbf{6 4 . 1}$ & $\mathbf{9 7 . 7}$ \\
\hline & MAD & & $\mathbf{6 4 . 5}$ & $\mathbf{3 8 . 5}$ & $\mathbf{3 2 . 9}$ & $\mathbf{4 5 . 2}$ & $\mathbf{2 2 . 2}$ & $\mathbf{1 6 . 7}$ \\
\hline & MAPE & & $\mathbf{4 . 5 2}$ & $\mathbf{3 . 6 5}$ & $\mathbf{2 . 8 6}$ & $\mathbf{2 . 6 6}$ & $\mathbf{1 . 8 7}$ & $\mathbf{1 . 0 6}$ \\
\hline
\end{tabular}


Table S4. Comparisons of harmonic (HO), VSCF and VSCF-PT2 values with experiment for fundamental transitions (in $\mathrm{cm}^{-1}$ ) computed at $\operatorname{CCSD}$ and $\operatorname{CCSD}(\mathrm{T})$ potentials for heptatomic molecules along with the statistical error analysis

\begin{tabular}{|c|c|c|c|c|c|c|c|c|}
\hline & Mode type & & \multicolumn{3}{|c|}{ CCSD } & \multicolumn{3}{|c|}{$\operatorname{CCSD}(\mathrm{T})$} \\
\hline & $\mathrm{C}_{2} \mathrm{H}_{4} \mathrm{O}$ & Exp & HO & VSCF & VSCF-PT2 & HO & VSCF & VSCF-PT2 \\
\hline$v_{1}$ & $\mathrm{CH} 2$ a-str & 3065 & 3232.2 & 3096.1 & 3082.0 & 3210.8 & 3071.5 & 3057.1 \\
\hline $\mathrm{v}_{2}$ & $\mathrm{CH} 2$ a-str & 3065 & 3217.5 & 3079.5 & 3065.0 & 3196.2 & 3054.9 & 3040.0 \\
\hline$v_{3}$ & $\mathrm{CH} 2$ s-str & 3024 & 3140.5 & 3040.5 & 2998.3 & 3117.4 & 3014.6 & 2969.4 \\
\hline$v_{4}$ & $\mathrm{CH} 2 \mathrm{~s}-\mathrm{str}$ & 2978 & 3131.4 & 3010.5 & 2997.8 & 3109.1 & 2985.2 & 2972.2 \\
\hline$v_{5}$ & $\mathrm{CH} 2$ scis & 1498 & 1570.6 & 1540.0 & 1530.1 & 1549.7 & 1517.3 & 1506.9 \\
\hline$v_{6}$ & $\mathrm{CH} 2$ scis & 1470 & 1529.1 & 1497.8 & 1496.2 & 1513.0 & 1480.6 & 1479.0 \\
\hline$v_{7}$ & Ring str & 1270 & 1324.2 & 1305.6 & 1297.1 & 1300.1 & 1280.0 & 1271.0 \\
\hline$v_{8}$ & $\mathrm{CH} 2$ wag & 1150 & 1193.1 & 1179.0 & 1176.4 & 1176.2 & 1165.5 & 1162.9 \\
\hline$v_{9}$ & $\mathrm{CH} 2$ twist & 1147 & 1190.5 & 1180.2 & 1177.6 & 1174.9 & 1160.1 & 1157.3 \\
\hline$v_{10}$ & $\mathrm{CH} 2$ wag & 1159 & 1182.8 & 1172.8 & 1163.3 & 1157.9 & 1147.5 & 1137.1 \\
\hline$v_{11}$ & $\mathrm{CH} 2$ twist & 1120 & 1178.9 & 1170.8 & 1167.3 & 1156.6 & 1148.0 & 1144.4 \\
\hline$v_{12}$ & $\mathrm{CH} 2$ twist & 1020 & 1068.2 & 1057.3 & 1053.4 & 1051.7 & 1040.8 & 1036.6 \\
\hline$v_{13}$ & ring str. & 877 & 920.6 & 905.7 & 899.0 & 899.7 & 883.5 & 876.2 \\
\hline$v_{14}$ & CO str. & 822 & 883.4 & 861.6 & 856.9 & 849.9 & 826.4 & 821.2 \\
\hline$v_{15}$ & $\mathrm{CH} 2$ rock & 808 & 826.5 & 834.2 & 827.0 & 815.8 & 823.5 & 816.2 \\
\hline & MaxAD & & 167.2 & 50.8 & 47.3 & 145.8 & 28.0 & 54.6 \\
\hline & MAD & & 74.4 & 30.6 & 24.4 & 53.9 & 12.6 & 13.9 \\
\hline & MAPE & & 4.46 & 2.45 & 2.00 & 2.89 & 1.01 & 0.88 \\
\hline & $\mathrm{C}_{2} \mathrm{H}_{2} \mathrm{~N}_{2} \mathrm{O}$ & & & & & & & \\
\hline$v_{1}$ & $\mathrm{CH}$ str & 3140 & 3315.5 & 3182.4 & 3144.4 & 3290.4 & 3154.1 & 3138.2 \\
\hline$v_{2}$ & $\mathrm{CH}$ str & 3133 & 3301.1 & 3116.2 & 3085.7 & 3275.7 & 3086.5 & 3055.3 \\
\hline$v_{3}$ & ip-Ring I & 1546 & 1626.8 & 1599.5 & 1595.4 & 1568.7 & 1539.5 & 1535.3 \\
\hline$v_{4}$ & ip-Ring II & 1418 & 1503.7 & 1480.0 & 1474.1 & 1456.4 & 1430.0 & 1423.7 \\
\hline$v_{5}$ & ip-Ring III & 1316 & 1371.4 & 1354.7 & 1349.0 & 1335.5 & 1318.0 & 1311.3 \\
\hline$v_{6}$ & CH ip-bend & 1177 & 1227.6 & 1210.8 & 1206.8 & 1198.8 & 1181.1 & 1176.8 \\
\hline$v_{7}$ & $\mathrm{CH}$ ip-bend & 1038 & 1070.8 & 1054.7 & 1051.2 & 1059.5 & 1041.7 & 1037.7 \\
\hline$v_{8}$ & ip-Ring IV & 1006 & 1052.2 & 1040.3 & 1034.4 & 1022.0 & 1008.1 & 1001.5 \\
\hline$v_{9}$ & ip-Ring V & 952 & 975.2 & 964.0 & 963.9 & 955.9 & 943.7 & 943.5 \\
\hline$v_{10}$ & ip-Ring VI & 889 & 957.9 & 926.1 & 920.2 & 899.6 & 903.1 & 891.5 \\
\hline$v_{11}$ & ip-Ring VII & 872 & 929.5 & 932.6 & 921.8 & 891.7 & 881.8 & 871.7 \\
\hline$v_{12}$ & $\mathrm{CH}$ op-bend & 839 & 921.6 & 912.7 & 904.8 & 880.1 & 842.6 & 835.4 \\
\hline$v_{13}$ & $\mathrm{CH}$ op-bend & 824 & 884.6 & 889.1 & 876.0 & 859.5 & 863.9 & 850.1 \\
\hline
\end{tabular}




\begin{tabular}{|l|l|c|c|c|c|c|c|c|}
\hline$v_{14}$ & op-Ring I & 635 & 661.7 & 655.7 & 654.3 & 646.8 & 639.7 & 638.5 \\
\hline$v_{15}$ & op-Ring II & 631 & 652.9 & 641.8 & 641.3 & 639.3 & 627.8 & 627.3 \\
\hline & MaxAD & & $\mathbf{1 7 5 . 5}$ & $\mathbf{7 3 . 7}$ & $\mathbf{6 5 . 8}$ & $\mathbf{1 5 0 . 4}$ & $\mathbf{4 6 . 5}$ & $\mathbf{7 7 . 7}$ \\
\hline & MAD & & $\mathbf{6 9 . 1}$ & $\mathbf{3 8 . 6}$ & $\mathbf{3 3 . 5}$ & $\mathbf{3 7 . 6}$ & $\mathbf{1 1 . 7}$ & $\mathbf{1 0 . 3}$ \\
\hline & MAPE & & $\mathbf{5 . 3 4}$ & $\mathbf{3 . 6 4}$ & $\mathbf{3 . 1 5}$ & $\mathbf{2 . 4 5}$ & $\mathbf{0 . 9 6}$ & $\mathbf{0 . 6 9}$ \\
\hline
\end{tabular}


Table S5. Comparison of different vibrational algorithms for the anharmonic transitions of $\mathrm{H}_{2} \mathrm{CO}$ (in $\mathrm{cm}^{-1}$ ).

\begin{tabular}{|c|c|c|c|c|c|c|c|c|}
\hline & Mode type & Ref. & \multicolumn{4}{|c|}{$\operatorname{CCSD}(\mathrm{T})$} & Ref. & \\
\hline & $\mathrm{H}_{2} \mathrm{CO}$ & Exp & VSCF & VSCF-PT2 & VSCF/VCI* & $\mathrm{VCI}^{\#}$ & $\mathrm{VCI}^{\#}$ & VSCF-PT2 \\
\hline$v_{1}$ & $\mathrm{CH} 2$ a-str & 2843 & 2806.9 & 2773.4 & 2824.56 & 2843.6 & 2843.6 & 2773.4 \\
\hline$v_{2}$ & $\mathrm{CH} 2$ s-str & 2783 & 2814.1 & 2766.7 & 2776.36 & 2783.4 & 2783.4 & 2766.7 \\
\hline$v_{3}$ & $\mathrm{CO}$ str & 1746 & 1751.7 & 1749.3 & 1767.5 & 1734.9 & 1734.9 & 1749.3 \\
\hline$v_{4}$ & $\mathrm{CH} 2$ scis & 1500 & 1509.2 & 1504.7 & 1523.63 & 1494.7 & 1494.7 & 1504.7 \\
\hline$v_{5}$ & $\mathrm{CH} 2$ rock & 1249 & 1249.8 & 1246.9 & 1267.46 & 1240.6 & 1240.6 & 1246.9 \\
\hline$v_{6}$ & $\mathrm{CH} 2$ wag & 1167 & 1159.5 & 1155.2 & 1177.28 & 1187.6 & 1187.6 & 1155.2 \\
\hline & MaxAD & & 36.1 & 69.6 & 23.63 & 20.6 & & 70.2 \\
\hline & MAD & & 15.1 & 18.0 & 16.49 & 7.73 & & 25.0 \\
\hline & MAPE & & 0.67 & 0.79 & 1.01 & 0.58 & & 1.3 \\
\hline
\end{tabular}

*VSCF/VCI uses the virtual CI solver on VSCF top of VSCF within a space of the ground state and both the singly and doubly excited vibrational levels as implemented in GAMESS.

${ }^{\#}$ State-specific configuration selective VCI calculations which included configurations from single up to quadruple excitations, VCI(SDTQ). (Rauhut et al. J. Chem. Phys. 130, 054105, (2009)).

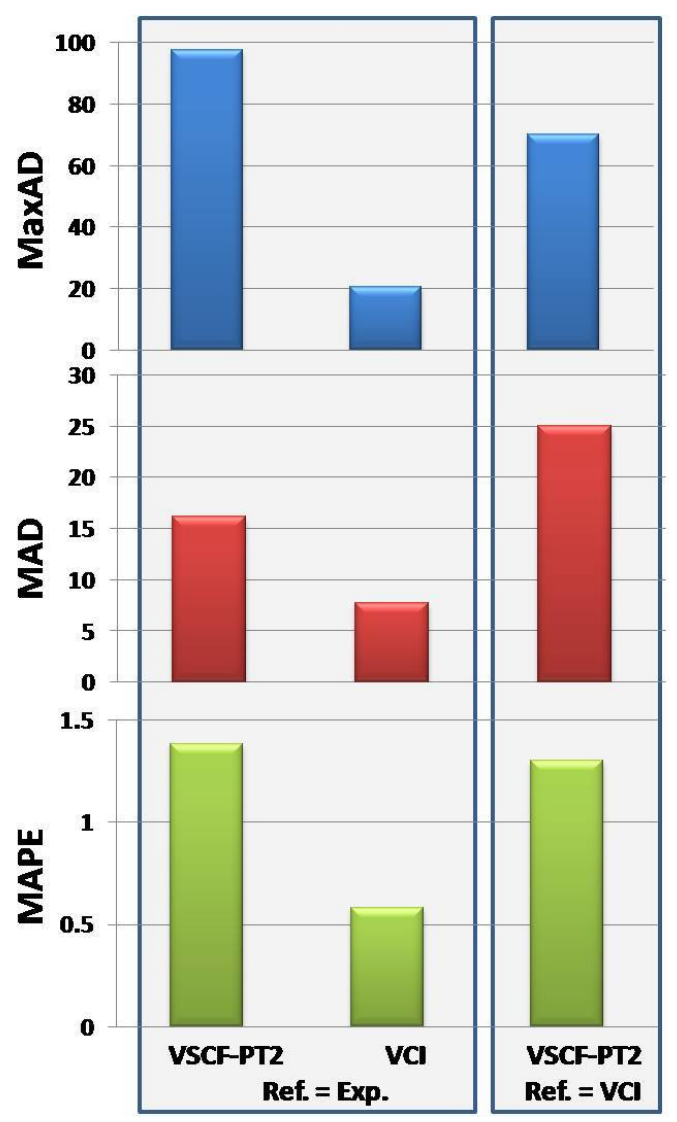

Fig. S2. Comparisons of the errors of VSCF-PT2 and VCI. In the left and right panels the reference values are experiment and VCI, respectively. Here VCI is VCI(SDTQ). 
Table S6. Comparisons of harmonic (HO), VSCF and VSCF-PT2 values with experiment for the fundamental transitions (in $\mathrm{cm}^{-1}$ ) computed at different $\mathrm{CC}$ level of theories along with the statistical error analysis.

\begin{tabular}{|c|c|c|c|c|c|c|c|c|c|c|c|}
\hline $\mathrm{H}_{2} \mathrm{O}$ & & \multicolumn{2}{|c|}{ CCD } & \multicolumn{2}{|c|}{ CCSD } & \multicolumn{2}{|c|}{ CR-CCL } & \multicolumn{2}{|c|}{$\operatorname{CCSD}(\mathrm{T})$} & \multicolumn{2}{|c|}{ CCSD(TQ) } \\
\hline & Exp & HO & $\begin{array}{c}\text { VSCF- } \\
\text { PT2 }\end{array}$ & HO & $\begin{array}{c}\text { VSCF- } \\
\text { PT2 }\end{array}$ & HO & $\begin{array}{c}\text { VSCF- } \\
\text { PT2 }\end{array}$ & HO & $\begin{array}{c}\text { VSCF- } \\
\text { PT2 }\end{array}$ & HO & $\begin{array}{c}\text { VSCF- } \\
\text { PT2 }\end{array}$ \\
\hline$v_{1}$ & 3756 & 3995.4 & 3783.3 & 3979.1 & 3763.3 & 3949.6 & 3730.5 & 3945.5 & 3725.7 & 3943.0 & 3722.6 \\
\hline$v_{2}$ & 3657 & 3892.9 & 3718.7 & 3876.0 & 3699.4 & 3845.5 & 3666.0 & 3841.0 & 3660.6 & 3837.9 & 3657.0 \\
\hline$v_{3}$ & 1595 & 1680.8 & 1614.6 & 1678.0 & 1612.4 & 1669.6 & 1603.8 & 1668.4 & 1602.6 & 1668.1 & 1602.3 \\
\hline $\operatorname{MaxAD}$ & & 239.4 & 61.7 & 223.1 & 42.4 & 193.6 & 25.5 & 189.5 & 30.3 & 187.0 & 33.4 \\
\hline MAD & & 187.0 & 36.2 & 175.0 & 22.4 & 152.2 & 14.4 & 149.0 & 13.9 & 147.0 & 13.6 \\
\hline MAPE & & 6.07 & 1.21 & 5.71 & 0.81 & 5.00 & 0.49 & 4.89 & 0.46 & 4.84 & 0.45 \\
\hline $\mathrm{H}_{2} \mathrm{CO}$ & & \multicolumn{2}{|c|}{ CCD } & \multicolumn{2}{|c|}{ CCSD } & \multicolumn{2}{|c|}{ CR-CCL } & \multicolumn{2}{|c|}{ CCSD(T) } & \multicolumn{2}{|c|}{ CCSD(TQ) } \\
\hline & Exp & HO & $\begin{array}{c}\text { VSCF- } \\
\text { PT2 }\end{array}$ & HO & $\begin{array}{c}\text { VSCF- } \\
\text { PT2 }\end{array}$ & HO & $\begin{array}{c}\text { VSCF- } \\
\text { PT2 }\end{array}$ & HO & $\begin{array}{c}\text { VSCF- } \\
\text { PT2 }\end{array}$ & HO & $\begin{array}{c}\text { VSCF- } \\
\text { PT2 }\end{array}$ \\
\hline$v_{1}$ & 2843 & 3039.2 & 2825.5 & 3026.6 & 2811.6 & 2997.9 & 2776.4 & 2996.2 & 2773.4 & 3000.9 & 2778.9 \\
\hline$v_{2}$ & 2783 & 2965.4 & 2810.5 & 2958.5 & 2801.8 & 2931.4 & 2769.6 & 2929.4 & 2766.7 & 2933.3 & 2771.2 \\
\hline$v_{3}$ & 1746 & 1852.8 & 1826.1 & 1829.5 & 1800.9 & 1788.6 & 1758.1 & 1780.8 & 1749.3 & 1780.6 & 1749.5 \\
\hline$v_{4}$ & 1500 & 1566.8 & 1529.1 & 1560.9 & 1523.1 & 1545.0 & 1507.2 & 1543.5 & 1504.7 & 1544.3 & 1505.9 \\
\hline$v_{5}$ & 1249 & 1298.0 & 1269.1 & 1289.8 & 1263.5 & 1276.7 & 1248.8 & 1274.2 & 1246.9 & 1275.2 & 1247.9 \\
\hline$v_{6}$ & 1167 & 1225.9 & 1184.9 & 1215.1 & 1179.3 & 1195.6 & 1159.3 & 1192.1 & 1155.2 & 1191.0 & 1154.1 \\
\hline MaxAD & & 196.2 & 80.1 & 183.60 & 54.90 & 154.9 & 66.6 & 153.2 & 69.6 & 157.9 & 64.1 \\
\hline MAD & & 110.0 & 32.0 & 98.8 & 25.8 & 74.5 & 17.9 & 71.4 & 18.0 & 72.9 & 16.6 \\
\hline MAPE & & 5.50 & 1.88 & 4.8 & 1.5 & 3.48 & 0.78 & 3.28 & 0.79 & 3.34 & 0.74 \\
\hline
\end{tabular}


Table S7: Comparisons of the VSCF-PT2 fundamental transitions (in $\mathrm{cm}^{-1}$ ) computed at CCSD(T) with cc-pVDZ and cc-pVTZ basis sets for $\mathrm{H}_{2} \mathrm{O}, \mathrm{H}_{2} \mathrm{CO}$ and $\mathrm{HCOOH}$ along the statistical error analysis.

\begin{tabular}{|c|c|c|c|c|c|c|}
\hline \multirow[t]{2}{*}{$\mathrm{H}_{2} \mathrm{O}$} & & & \multicolumn{2}{|c|}{ cc-pVDZ } & \multicolumn{2}{|c|}{ cc-pVTZ } \\
\hline & & Exp & HO & VSCF-PT2 & HO & VSCF-PT2 \\
\hline$v_{1}$ & $\mathrm{OH}$ a st & 3756 & 3927.59 & 3700.51 & 3945.5 & 3725.68 \\
\hline$v_{2}$ & $\mathrm{OH}$ s st & 3657 & 3821.66 & 3628.81 & 3841.0 & 3660.64 \\
\hline \multirow[t]{4}{*}{$v_{3}$} & Bend & 1595 & 1689.84 & 1622.96 & 1668.4 & 1602.59 \\
\hline & MaxAD & & 171.59 & 55.49 & 189.5 & 30.32 \\
\hline & MAD & & 143.7 & 37.21 & 149.0 & 13.85 \\
\hline & MAPE & & 5.01 & 1.33 & 4.89 & 0.46 \\
\hline $\mathrm{H}_{2} \mathrm{CO}$ & & Exp & HO & VSCF-PT2 & HO & VSCF-PT2 \\
\hline$v_{1}$ & $\mathrm{CH} 2 \mathrm{a}-\mathrm{str}$ & 2843 & 2986.72 & 2760.43 & 2996.2 & 2773.4 \\
\hline$v_{2}$ & $\mathrm{CH} 2 \mathrm{~s}-\mathrm{str}$ & 2783 & 2924.53 & 2754.64 & 2929.4 & 2766.7 \\
\hline$v_{3}$ & $\mathrm{CO}$ str & 1746 & 1786.48 & 1754.92 & 1780.8 & 1749.3 \\
\hline $\mathrm{v}_{4}$ & $\mathrm{CH} 2$ scis & 1500 & 1534.58 & 1496.33 & 1543.5 & 1504.7 \\
\hline$v_{5}$ & $\mathrm{CH} 2$ rock & 1249 & 1265.91 & 1238.51 & 1274.2 & 1246.9 \\
\hline \multirow[t]{4}{*}{$v_{6}$} & $\mathrm{CH} 2$ wag & 1167 & 1175.58 & 1137.8 & 1192.1 & 1155.2 \\
\hline & MaxAD & & 143.72 & 82.57 & 153.2 & 69.6 \\
\hline & MAD & & 64.3 & 27.2 & 71.4 & 18.0 \\
\hline & MAPE & & 2.81 & 1.34 & 3.28 & 0.79 \\
\hline НСООН & & Exp & HO & VSCF-PT2 & HO & VSCF-PT2 \\
\hline$v_{1}$ & $\mathrm{OH}$ str & 3570 & 3744.46 & 3525.38 & 3763.3 & 3547.1 \\
\hline$v_{2}$ & $\mathrm{CH}$ str & 2943 & 3082.88 & 2920.19 & 3089.2 & 2932.5 \\
\hline$v_{3}$ & $\mathrm{C}=\mathrm{O}$ str & 1770 & 1843.96 & 1807.24 & 1824.6 & 1789.5 \\
\hline$v_{4}$ & $\mathrm{CH}$ bend & 1387 & 1414.38 & 1374.85 & 1415.7 & 1375.5 \\
\hline$v_{5}$ & $\mathrm{OH}$ bend & 1229 & 1334.64 & 1294.99 & 1326.0 & 1284.6 \\
\hline$v_{6}$ & $\mathrm{C}-\mathrm{O}$ str & 1105 & 1147.35 & 1102.33 & 1137.1 & 1090.4 \\
\hline$v_{7}$ & $\mathrm{CH}$ bend & 1033 & 1053.71 & 1022.59 & 1061.0 & 1030.9 \\
\hline$v_{8}$ & Torsion & 638 & 692.72 & 616.98 & 676.6 & 598.2 \\
\hline \multirow[t]{4}{*}{$v_{9}$} & OCO deform & 625 & 629.51 & 618.24 & 629.4 & 618.0 \\
\hline & MaxAD & & 174.46 & 65.99 & 193.3 & 55.6 \\
\hline & MAD & & 71.51 & 24.85 & 69.2 & 20.4 \\
\hline & MAPE & & 4.39 & 1.78 & 3.98 & 1.82 \\
\hline
\end{tabular}


Table S8. Comparisons of the VSCF-PT2 values computed at CCSD(T) method using pure and hybrid (cc-pVQZ /cc-pVTZ) PES with experimental values (in $\mathrm{cm}^{-1}$ )

\begin{tabular}{|c|c|c|c|c|c|c|c|}
\hline & & & & VTZ & & VQZ & Hybrid \\
\hline & $\mathrm{H}_{2} \mathrm{O}$ & $\operatorname{Exp}$ & HO & VSCF-PT2 & HO & VSCF-PT2 & VSCF-PT2 \\
\hline$v_{1}$ & $\mathrm{OH}$ a st & 3756 & 3945.5 & 3725.7 & 3951.53 & 3730.91 & 3731.32 \\
\hline $\mathrm{v}_{2}$ & $\mathrm{OH}$ s st & 3657 & 3841.0 & 3660.6 & 3844.39 & 3663.27 & 3657.16 \\
\hline$v_{3}$ & Bend & 1595 & 1668.4 & 1602.6 & 1658.74 & 1591.29 & 1593.36 \\
\hline & MaxAD & & 189.5 & 30.3 & 195.53 & 25.09 & 24.68 \\
\hline & MAD & & 149.0 & 13.9 & 148.89 & 11.69 & 8.83 \\
\hline & MAPE & & 4.89 & 0.46 & 4.78 & 0.36 & 0.25 \\
\hline & $\mathrm{H}_{2} \mathrm{CO}$ & $\operatorname{Exp}$ & HO & VSCF-PT2 & HO & VSCF-PT2 & VSCF-PT2 \\
\hline$\overline{v_{1}}$ & $\mathrm{CH} 2$ a-str & 2843 & 2996.2 & 2773.4 & 3004.21 & 2780.51 & 2770.89 \\
\hline$v_{2}$ & $\mathrm{CH} 2$ s-str & 2783 & 2929.4 & 2766.7 & 2933.73 & 2769.58 & 2750.07 \\
\hline$v_{3}$ & $\mathrm{CO}$ str & 1746 & 1780.8 & 1749.3 & 1781.38 & 1750.43 & 1742.6 \\
\hline$v_{4}$ & $\mathrm{CH} 2$ scis & 1500 & 1543.5 & 1504.7 & 1537.55 & 1499.38 & 1506.57 \\
\hline$v_{5}$ & $\mathrm{CH} 2$ rock & 1249 & 1274.2 & 1246.9 & 1273.22 & 1244.83 & 1248.77 \\
\hline$v_{6}$ & $\mathrm{CH} 2$ wag & 1167 & 1192.1 & 1155.2 & 1190.97 & 1154.12 & 1158.42 \\
\hline & MaxAD & & 153.2 & 69.6 & 161.21 & 62.49 & 72.11 \\
\hline & MAD & & 71.4 & 18.0 & 72.18 & 16.34 & 20.64 \\
\hline & MAPE & & 3.28 & 0.79 & 3.27 & 0.74 & 0.85 \\
\hline
\end{tabular}


S9. Cartesian coordinates of equilibrium geometries of some selected molecules.

\section{A. Molecule: $\mathrm{H}_{2} \mathrm{O}$}

\section{Method: CCSD}

$\begin{array}{lllll}\mathrm{O} & 8.0 & 0.0000000000 & 0.0000000000 & 0.2176422368 \\ \mathrm{H} & 1.0 & -0.0000000000 & -1.4242285557 & -0.8972629885 \\ \mathrm{H} & 1.0 & 0.0000000000 & 1.4242285557 & -0.8972629885\end{array}$

\section{Method: CCSD(T)}

$\begin{array}{lllll}\mathrm{O} & 8.0 & 0.0000000000 & 0.0000000000 & 0.2220017018 \\ \mathrm{H} & 1.0 & -0.0000000000 & -1.4246371313 & -0.8994427209 \\ \mathrm{H} & 1.0 & 0.0000000000 & 1.4246371313 & -0.8994427209\end{array}$

\section{B. Molecule: $\mathrm{F}_{2} \mathbf{C O}$}

Method: CCSD

$\begin{array}{llllr}\text { C } & 6.0 & 0.0000000000 & 0.0000000000 & 0.2651814057 \\ \text { O } & 8.0 & 0.0000000000 & 0.0000000000 & 2.4772780528 \\ \text { F } & 9.0 & 0.0000000000 & 1.9979968784 & -1.1872592352 \\ \text { F } & 9.0 & 0.0000000000 & -1.9979968784 & -1.1872592352\end{array}$

\section{Method: $\operatorname{CCSD}(\mathrm{T})$}

$\begin{array}{lllll}\mathrm{C} & 6.0 & 0.0000000000 & 0.0000000000 & 0.2671966276 \\ \mathrm{O} & 8.0 & -0.0000000000 & 0.0000000000 & 2.4897156474 \\ \mathrm{~F} & 9.0 & 0.0000000000 & 2.0057917101 & -1.1944856433 \\ \mathrm{~F} & 9.0 & 0.0000000000 & -2.0057917101 & -1.1944856433\end{array}$

\section{Molecule: $\mathrm{HCOOH}$}

\section{Method: CCSD}

$\begin{array}{lllll}\mathrm{O} & 8.0 & -0.0252358177 & -0.0108323383 & 0.0000000000 \\ \mathrm{C} & 6.0 & 2.2328149783 & 0.0957443913 & 0.0000000000 \\ \mathrm{O} & 8.0 & 3.5886301537 & 2.2370431686 & 0.0000000000 \\ \mathrm{H} & 1.0 & 2.3919340809 & 3.6137163373 & 0.0000000000 \\ \mathrm{H} & 1.0 & 3.4967882770 & -1.5363894590 & 0.0000000000\end{array}$

\section{Method: CCSD(T)}

$\begin{array}{lllll}\mathrm{O} & 8.0 & -0.0361773943 & -0.0099063248 & 0.0000000000 \\ \mathrm{C} & 6.0 & 2.2333606065 & 0.0932660156 & 0.0000000000 \\ \mathrm{O} & 8.0 & 3.5987333768 & 2.2419051974 & 0.0000000000 \\ \mathrm{H} & 1.0 & 2.3875621397 & 3.6144167587 & 0.0000000000 \\ \mathrm{H} & 1.0 & 3.5014529435 & -1.5403995475 & 0.0000000000\end{array}$

\section{Molecule:}




\begin{tabular}{lcccc}
\multicolumn{6}{l}{ Method: CCSD } \\
C & 6.0 & -0.0000000000 & -1.3448745834 & -1.6617244928 \\
$\mathrm{C}$ & 6.0 & -0.0000000000 & 1.3448745834 & -1.6617244928 \\
$\mathrm{H}$ & 1.0 & -0.0000000000 & -2.6576486208 & -3.2106473170 \\
$\mathrm{H}$ & 1.0 & 0.0000000000 & 2.6576486208 & -3.2106473170 \\
$\mathrm{~N}$ & 7.0 & -0.0000000000 & -2.1242689324 & 0.6649644997 \\
$\mathrm{~N}$ & 7.0 & -0.0000000000 & 2.1242689324 & 0.6649644997 \\
$\mathrm{O}$ & 8.0 & 0.0000000000 & 0.0000000000 & 2.1149995494
\end{tabular}

\section{Method: CCSD(T)}

$\begin{array}{lcccc}\mathrm{C} & 6.0 & -0.0000000000 & -1.3443487611 & -1.6680102328 \\ \mathrm{C} & 6.0 & 0.0000000000 & 1.3443487611 & -1.6680102328 \\ \mathrm{H} & 1.0 & -0.0000000000 & -2.6574081337 & -3.2218205487 \\ \mathrm{H} & 1.0 & 0.0000000000 & 2.6574081337 & -3.2218205487 \\ \mathrm{~N} & 7.0 & -0.0000000000 & -2.1438792169 & 0.6727859386 \\ \mathrm{~N} & 7.0 & 0.0000000000 & 2.1438792169 & 0.6727859386 \\ \mathrm{O} & 8.0 & 0.0000000000 & 0.0000000000 & 2.1342746148\end{array}$


S10. Source and references of experimental data for each molecule

\begin{tabular}{|c|c|c|}
\hline \multicolumn{3}{|c|}{ Fundamentals } \\
\hline S. No. & Molecule(s) & References \\
\hline 1 & $\begin{array}{l}\mathrm{H}_{2} \mathrm{O}, \mathrm{F}_{2} \mathrm{O}, \mathrm{HOF}, \mathrm{HNO}, \\
\mathrm{F}_{2} \mathrm{CO}, \mathrm{HOCN}, \text { trans-HNCO, } \\
\mathrm{HCOOH}, \quad \mathrm{CH}_{2} \mathrm{NH}, \quad \mathrm{C}_{2} \mathrm{H}_{4}, \\
\mathrm{C}_{2} \mathrm{H}_{2} \mathrm{O}_{2}, \mathrm{C}_{2} \mathrm{H}_{4} \mathrm{O}, \mathrm{C}_{2} \mathrm{H}_{2} \mathrm{~N}_{2} \mathrm{O}\end{array}$ & $\begin{array}{l}\text { Gas Phase data from NIST data base (Shimanouchi, } \\
\text { T., Tables of Molecular Vibrational Frequencies } \\
\text { Consolidated Volume I, National Bureau of Standards, } \\
\text { 1972, 1-160) }\end{array}$ \\
\hline 2 & $\mathrm{H}_{2} \mathrm{CCO}$ & J. Chem. Phys. 102, 8506 (1995) \\
\hline 3 & $1,1-\mathrm{C}_{2} \mathrm{H}_{2} \mathrm{~F}_{2}$ & J. Phys. Chem. A 114, 5728 (2010) \\
\hline \multicolumn{3}{|c|}{ Excited states } \\
\hline 1 & $\mathrm{H}_{2} \mathrm{O}$ & J. Mol. Spectro. 133, 438 (1989) \\
\hline 2 & HOF & $\begin{array}{l}\text { J. Mol. Spectro. 136, } 197 \text { (1989) } \\
\text { J. Mol. Spectro. 138, } 346 \text { (1989) }\end{array}$ \\
\hline 3 & $\mathrm{H}_{2} \mathrm{CO}$ & $\begin{array}{l}\text { J. Chem. Phys. 82, } 9 \text { (1985) } \\
\text { J Chem. Phys. 118, } 1653 \text { (2003) }\end{array}$ \\
\hline 4 & $\mathbf{C}_{2} \mathbf{H}_{4}$ & Mol. Phys. 97, 265 (1999) \\
\hline 5 & НСООН & Chemical Physics, 283, 47 (2002) \\
\hline 6 & H2C2O & Spectr. Acta A, 67, 870 (2007) (and references therein) \\
\hline \multicolumn{3}{|c|}{ Intensities } \\
\hline 1 & $\mathrm{H}_{2} \mathrm{CO}$ & $\begin{array}{l}\text { J. Chem. Phys. 76, } 3860 \text { (1982) } \\
\text { Chem. Phys. Lett. 380, } 237 \text { (2003) }\end{array}$ \\
\hline 2 & $\mathbf{C}_{2} \mathbf{H}_{4}$ & J. Chem. Phys. 70, 2471, (1979) \\
\hline 3 & H2C2O & J. Chem. Phys. 74, 5384, (1981) \\
\hline 4 & DFE & J Mol Struct. 1009, 49 (2012) \\
\hline
\end{tabular}

